\title{
IUCrJ
}

Volume 2 (2015)

Supporting information for article:

Second harmonic generation from the "centrosymmetric" crystals Venkatram Nalla, Raghavender Medishetty, Wang Yue, Zhaozhi Bai, Nikolay I. Zheludev, Sun Handong, Ji Wei and Jagadese J. Vittal 


\section{Second harmonic generation from the "centrosymmetric" crystals}

\section{Venkatram Nalla ${ }^{\mathrm{a}}$, Raghavender Medishetty ${ }^{\mathrm{b}}$, Wang Yue ${ }^{\mathrm{c}}$, Zhaozhi Bai ${ }^{\mathrm{b}}$, Handong Sun ${ }^{\mathrm{a}^{*}}$, Wei}

$\mathbf{J i}^{\mathrm{d} *}$ and Jagadese $\mathbf{J}$. Vittal ${ }^{\mathrm{b} *}$

${ }^{a}$ Centre for Disruptive Photonic Technologies, Nanyang Technological University, Singapore, 637371 ${ }^{b}$ Department of Chemistry, National University of Singapore, 3 Science Drive 3, Singapore 117543 'Division of Physics \& Applied Physics, School of Physical \& Mathematical Sciences, Nanyang Technological University, Singapore 637371

dDepartment of Physics, National University of Singapore, 3 Science Drive 3, Singapore, Singapore 117542

\section{Supporting information}

The crystal data were refined purposely in the space group $C c$ to show the presence of both noncentrosymmetric and centrosymmetric packing in the crystal. The crystal was originally refined in the space group C2/c before (Medishetty, 2014, CCDC No. 979138).

Table S1 Cell data for 1 at different temperatures

\begin{tabular}{|c|c|c|}
\hline & 1 (E219) & $1(\mathrm{E} 210)$ \\
\hline Temperature (K) & $170(2)$ & $295(2)$ \\
\hline Sp. Grp & $C c$ & $C c$ \\
\hline$a(\AA)$ & $24.746(2)$ & $24.843(2)$ \\
\hline$b(\AA)$ & $12.212(1)$ & $12.245(1)$ \\
\hline$c(\AA)$ & $15.653(1)$ & $15.720(1)$ \\
\hline$\beta$ (deg) & $109.192(1)$ & $109.498(2)$ \\
\hline$V\left(\AA^{3}\right)$ & $4467.2(6)$ & $4507.9(7)$ \\
\hline$Z$ & 4 & 4 \\
\hline$D_{\text {calc }}\left(\mathrm{g} / \mathrm{cm}^{3}\right)$ & 1.454 & 1.440 \\
\hline$\mu\left(\mathrm{mm}^{-1}\right)$ & 1.134 & 1.124 \\
\hline GOF & 1.046 & 1.010 \\
\hline$R_{I}(I>2 \sigma(I))$ & 0.0323 & 0.0371 \\
\hline$w R_{2}(I>2 \sigma(I))$ & 0.0845 & 0.0898 \\
\hline
\end{tabular}

Only selected data are shown here since they are very similar and no trend has been found. Other data can be retrieved: CCDC 1031432-1031436 
Table S2 The Flack parameters for $\mathbf{1}$ obtained for two single crystals at different temperatures.

\begin{tabular}{|l|l|l|l|}
\hline Temperature & $\begin{array}{l}\text { SHG } \\
\text { found }\end{array}$ & $\begin{array}{l}\text { Flack parameter for the Crystal } \\
\text { showing High SHG }\end{array}$ & $\begin{array}{l}\text { Flack parameter for the Crystal } \\
\text { showing Low SHG }\end{array}$ \\
\hline $170 \mathrm{~K}$ & low & $0.34(3)$ & $0.38(3)$ \\
\hline $295 \mathrm{~K}$ & Medium & $0.10(3)$ & $0.41(3)$ \\
\hline $80 \mathrm{~K}$ & high & - & $0.37(3)$ \\
\hline
\end{tabular}

The Flack and Hooft parameters were also obtained using OLEX2 but the parameters changed and no correlation was found.

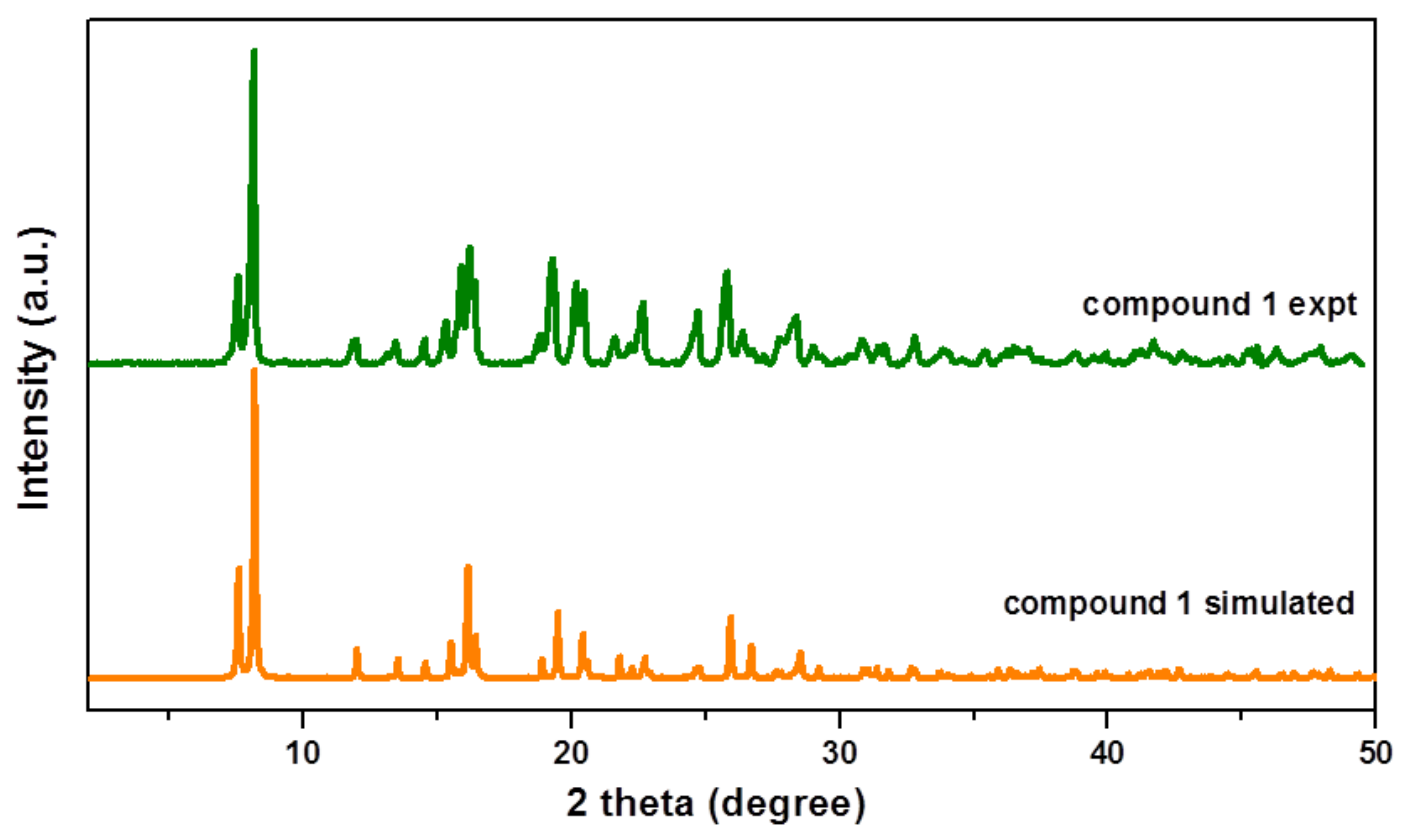

Figure S1 PXRD pattern of 1.

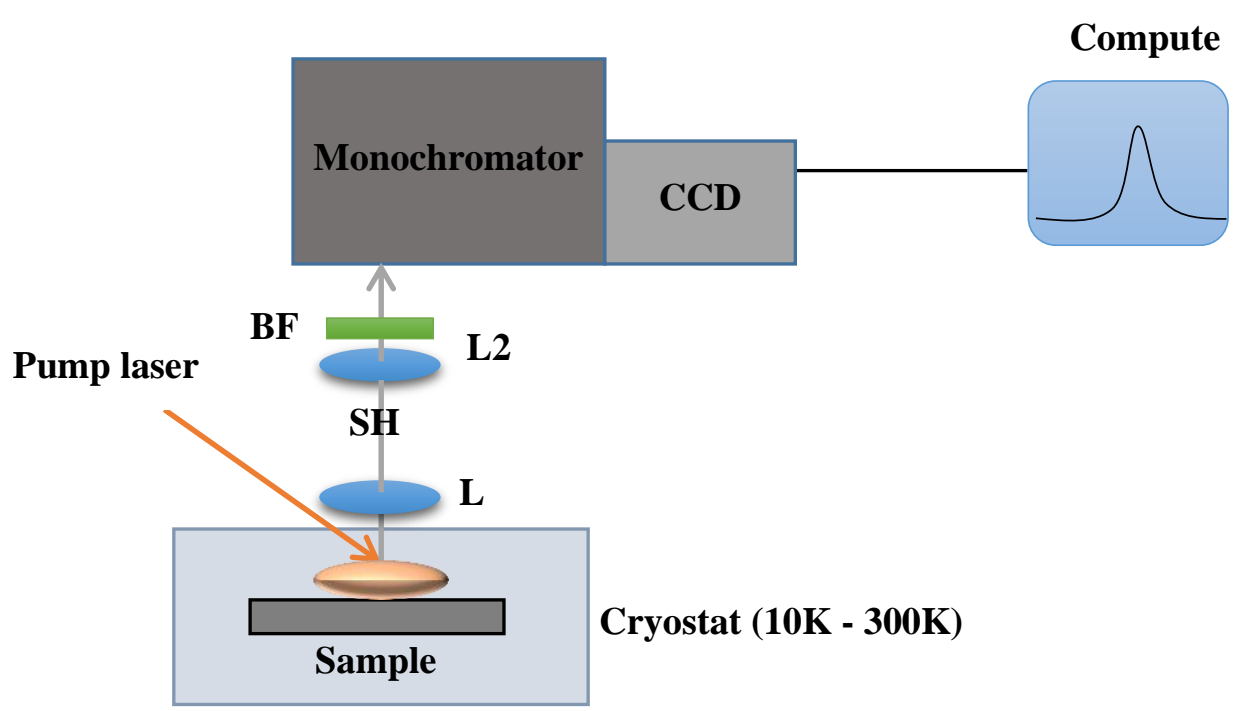

Figure S2 Schematic view of SHG measurements. 


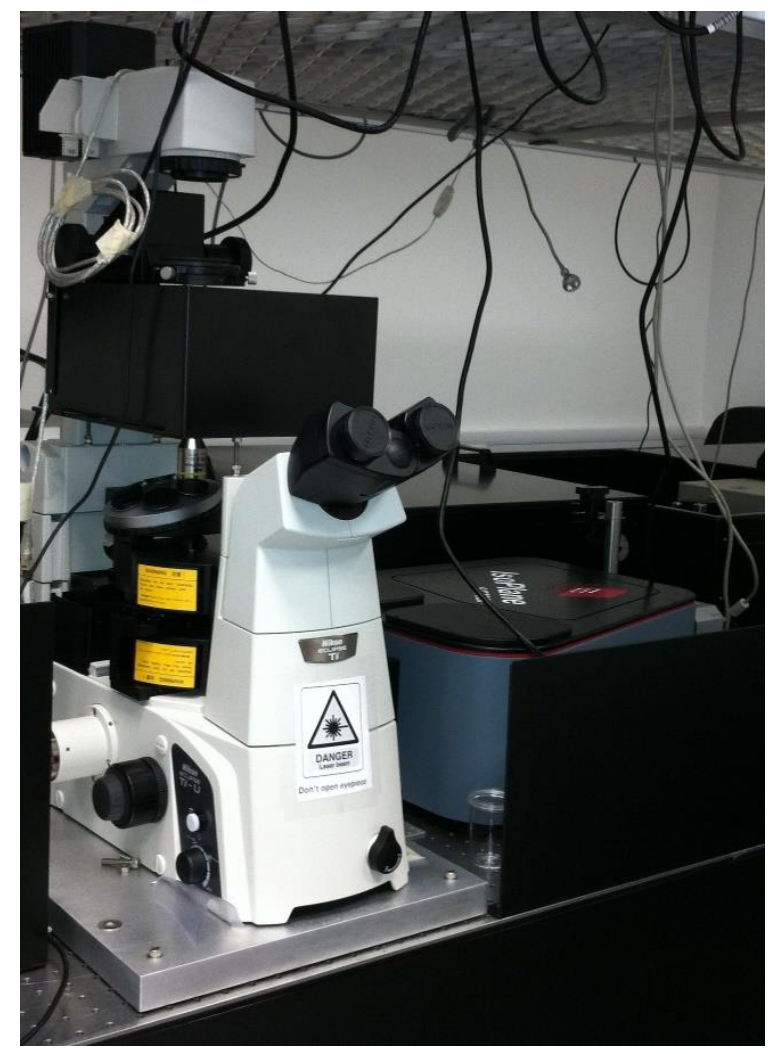

Figure S3 Experimental microscope set up for SHG measurement at room temperature.

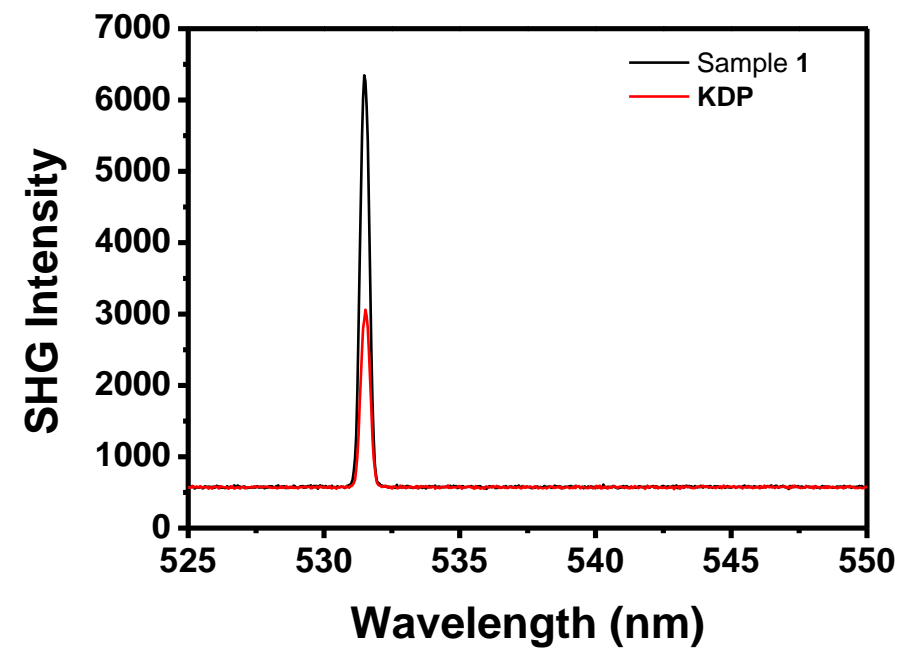

Figure S4 SHG measurement of KDP and $\mathbf{1 .}$ 


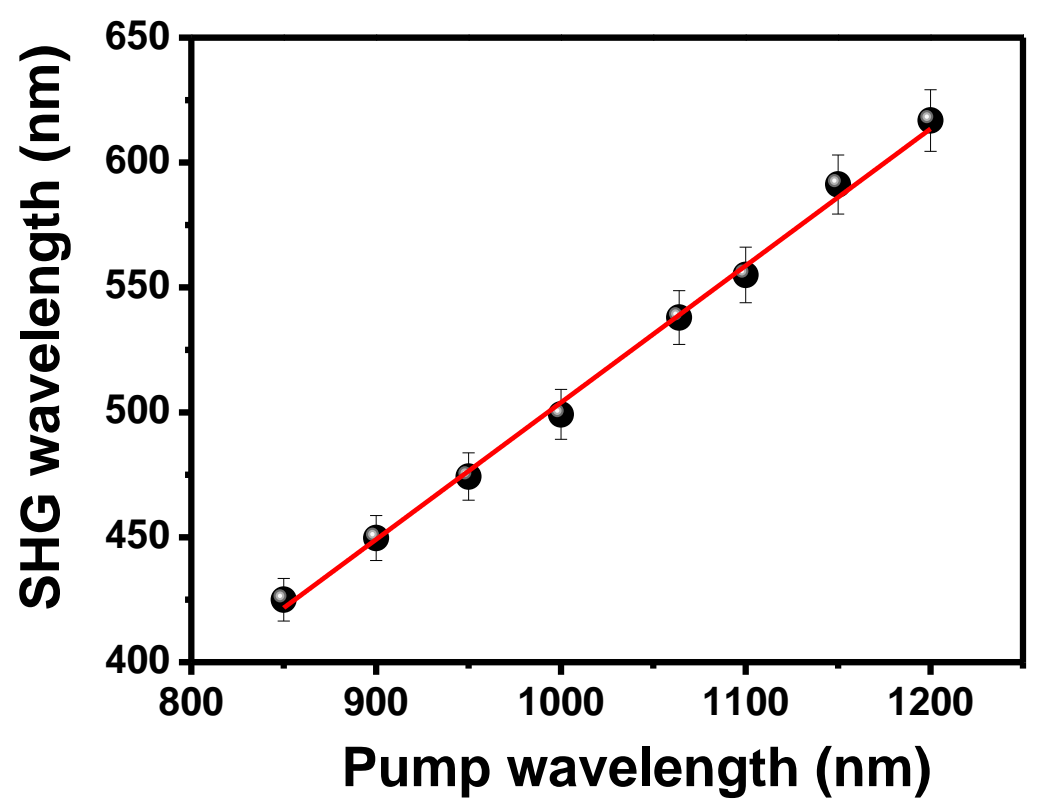

Figure S5 Excitation wavelength dependent SHG wavelength measurement of $\mathbf{1}$.

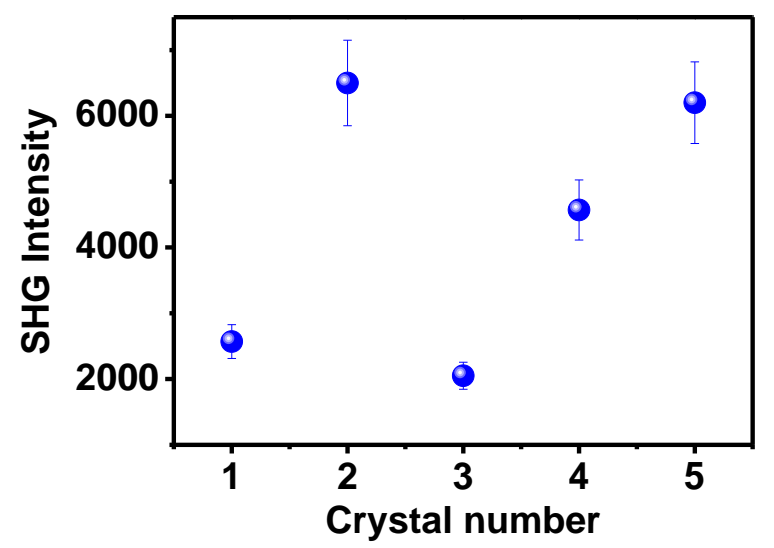

Figure S6 SHG intensities of different crystals from the same batch of $\mathbf{1}$. 